\title{
Algorithmic Manifold and Space Complexity
}

\author{
Takuya Yabu(takuya.yabu@live.jp)
}

\section{Introduction}

In the previous paper [1] [2], algorithmic manifolds were applied to the time complexity and discussed.

In this paper, I define algorithmic manifolds expressing space complexity and discuss topological properties. I also discuss the relationship between non-deterministic space complexity problems and deterministic Turing machines.

2. Algorithmic manifold of polynomial space

Think about the Riemannian manifold defined by the following metric.

$$
d s^{2}=d t^{2}+O\left(n^{k\left(l_{n}\right)}\right) d l_{n}^{2}
$$

Here, $t$ is the algorithm calculation time, $n$ is the data capacity to be input to the Turing machine to be processed, and $l_{n}$ is the data length of the algorithm of the Turing machine to be processed, and $O\left(n^{k\left(l_{n}\right)}\right)$ is the space complexity of the algorithm. The Riemannian manifold defined by the above metric is an algorithmic manifold representing an algorithm of polynomial space.

From equation ( 1 ), the Gauss curvature $K$ is given by the following equation.

$$
K=0
$$

That is, the algorithmic manifold of polynomial space is a flat plane. According to the same idea as in the previous paper [1] [2], PSPACE and NPSPACE can be said to be homeomorphic. That is

$$
\text { PSPACE }=\text { NPSPACE }
$$

Also, arbitrary two points on the algorithm manifold represented by the equation ( 1) are connected by a straight line. Therefore, as for PSPACE and NPSPACE as well, it is possible to convert into the problem of deterministic Turing machines, since straight lines can be determined by expressing the start point and end point of the algorithm on the algorithmic manifold expressed by equation (1). Also from this point, equation ( 3 ) holds.

3. Algorithmic manifold of exponential space

Think about the Riemannian manifold defined by the following metric.

$$
d s^{2}=d t^{2}+O\left(2^{n^{k\left(l_{n}\right)}}\right) d l_{n}^{2}
$$


Here, $O\left(2^{n^{k\left(l_{n}\right)}}\right)$ is the space complexity of the algorithm. The Riemannian manifold defined by the above metric is an algorithmic manifold representing an algorithm of exponential space.

From equation ( 4 ), the Gauss curvature $K$ is given by the following equation.

$$
K=0
$$

That is, the algorithmic manifold of exponential space is a flat plane. According to the same idea as in the previous paper [1] [2], EXPSPACE and NEXPSPACE can be said to be homeomorphic. That is

$$
\text { EXPSPACE }=\text { NEXPSPACE }
$$

Also, arbitrary two points on the algorithm manifold represented by the equation (4) are connected by a straight line. Therefore, as for EXPSPACE and NEXPSPACE as well, it is possible to convert into the problem of deterministic Turing machines, since straight lines can be determined by expressing the start point and end point of the algorithm on the algorithmic manifold expressed by equation (4). Also from this point, equation (6) holds.

4. Algorithmic manifold of logarithmic space

Think about the Riemannian manifold defined by the following metric.

$$
d s^{2}=d t^{2}+O\left(\log _{2} n\right) d l_{n}^{2}
$$

Here, $O\left(\log _{2} n\right)$ is the space complexity of the algorithm. The Riemannian manifold defined by the above metric is an algorithmic manifold representing an algorithm of logarithmic space.

From equation ( 7), the Gauss curvature $K$ is given by the following equation.

$$
K=0
$$

That is, the algorithmic manifold of logarithmic space is a flat plane. According to the same idea as in the previous paper [1] [2], L and NL can be said to be homeomorphic. That is

$$
\mathrm{L}=\mathrm{NL}
$$

Also, arbitrary two points on the algorithm manifold represented by the equation ( 7) are connected by a straight line. Therefore, as for L and NL as well, it is possible to convert into the problem of deterministic Turing machines, since straight lines can be determined by expressing the start point and end point of the algorithm on the algorithmic manifold expressed by equation ( 7). Also from this point, equation (9) holds. 


\section{Conclusion}

I define algorithmic manifolds expressing space complexity and clarify topological properties derived from them. I also clarified that non-deterministic space complexity problem can be described by deterministic Turing machine.

References

[1] T. Yabu, "Algorithmic Manifold and Application to P versus NP Problem," IEICE Transactions on Information and Systems, in Posting, https://osf.io/43jcw/, 2016.

[2] T. Yabu, “Application of Algorithmic Manifold to Exponential Time," IEICE Transactions on Information and Systems, in Posting, https://osf.io/ukg4g/, 2017. 